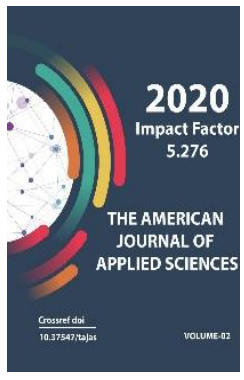

Copyright: Original content from this work may be used under the terms of the creative commons attributes 4.0 licence.

\section{Low And Highly Substituted Carboxymethyl Cellulose Synthesis From Powdered And Microcrystalline Cellulose}

\author{
Yuldoshov Sherzod Abdullaevich \\ Institute Of Polymer Chemistry And Physics Academy Of Sciences Of Uzbekistan, Tashkent, \\ Uzbekistan
}

Sarymsakov Abdushkur Abdukhalilovich

Institute Of Polymer Chemistry And Physics Academy Of Sciences Of Uzbekistan, Tashkent, Uzbekistan

\title{
ABSTRACT
}

The conditions for low and highly substituted water-soluble carboxymethyl cellulose synthesis based on microcrystalline and powder cellulose by the suspension method have been investigated. Accordingly, it was obtained CMC water-soluble samples with $0.38-0.40$ substitution degree based on microcrystalline and powder cellulose. And at one stage, there were obtained CMC samples with a high degree of substitution (1.4) by carboxymethylation so called polyanionic cellulose.

\section{KEYWORDS}

Microcrystalline cellulose, powdered cellulose, carboxymethyl cellulose, esterification, degree of substitution.

\section{INTRODUCTION}

Carboxymethyl cellulose (CMC) is an alkaline cellulose interaction product with monochloracetic acid or its sodium salt [1-5].

The CMC production from cellulose includes two stages chemical reactions [6-7]. The first stage is alkaline cellulose treatment and the second stage is the alkaline cellulose carboxymethylation reaction with an alkylating reagent.

Depending on feedstock, production technology type, CMC is produced on an industrial scale, which degree of substitution 
(DS) and degree of polymerization (DP). The currently produced $C M C$ has above $D S=0.6$ and $\mathrm{DP}=400[8,9]$.

Depending on carboxymethyl group number, CMC grades are subdivided into low $(\geq 0,5)$, medium $(0,55-0,85)$ and highly substituted $(\leq 0,9)$ grades. The $C M C$ samples with more than 0,9 substitution degree are called polyanionic cellulose [10-12]. Traditional CMC acquires the ability to dissolve in water with above DS $=0,5$ substitution degree.

We have investigated obtaining low- and highly-substituted water-soluble CMC possibilities samples based on microcrystalline cellulose (MCC) and powdered cellulose (PC) by the suspension method.

This method makes it possible to obtain products with a high degree substitution and uniform distribution of carboxymethyl groups along the cellulose macromolecule, which leads to an improvement in the CMC physicochemical and operational properties.

\section{MATERIALS AND METHODS}

\subsection{Microcrystalline cellulose}

MCC obtained by cotton cellulose acid hydrolysis produced by "Medikot" with the following quality indicators was used as a starting material for the CMC synthesis:

- $\quad$ Degree of crystallinity is $83 \%$;

- Degree of polymerization is 280 ;

- Particle size is $100 \mu \mathrm{m}$

\subsection{Powdered cellulose}

PCs obtained by cotton cellulose mechanical processing in a laboratory ball mill with the following quality indicators were also used as a feedstock for the CMC synthesis:

- degree of crystallinity is $22 \%$;

- degree of polymerization is 420 ;
- particle size is $100 \mu \mathrm{m}$.

2.3 Carboxymethyl cellulose synthesis

$10 \mathrm{~g}$ of the starting cellulose-containing raw material is treated with $60 \mathrm{ml}$ of ethyl alcohol and dispersed within 15 minutes. $\mathrm{A} 40 \% \mathrm{NaOH}$ solution is added with stirring. The alkaline treatment process is carried out for 90 minutes at $16^{\circ} \mathrm{C}$ temperature. Then add monochloracetic acid solution (MCAA) dissolved in $60 \mathrm{ml}$ ethyl alcohol by vigorous stirring for 15 minutes at $16^{\circ} \mathrm{C}$. After that, the reaction mixture temperature rises to $55^{\circ} \mathrm{C}$ and the esterification reaction is carried out for 3 hours. The product is filtered off on a glass filter, washed with aqueous ethanol. The product is dried at $60-70^{\circ} \mathrm{C}$ to a certain humidity.

\subsection{Characterization}

\subsubsection{CMC yield measurement}

CMC yield was measured based on a dry weight basis. The dried CMC net weight was divided by cellulose weight to get the yield value [13], as follow:

$$
\begin{gathered}
\text { CMC yield (\%) } \\
=\frac{\text { Weight of prepared CMC }(\mathrm{g})}{\text { Weight of driyed cellulose }(\mathrm{g})} \times 100 \\
\text { 2.4.2. Degree of substitution determination }
\end{gathered}
$$

\subsubsection{Degree of substitution determination}

To determine DS, $0.5 \mathrm{~g}$ of dried sodium CMC was ashed gently between 450 and $550^{\circ} \mathrm{C}$ for $24 \mathrm{~h}$, and then dissolved in $100 \mathrm{~mL}$ distilled water. $20 \mathrm{~mL}$ of this solution was titrated with $0.1 \mathrm{~N}$ sulphuric acid using methyl red as an indicator. After the first end point, the solution was boiled and titrated to a sharp end point. The carboxymethyl content was calculated from substitution degree [14], as follow: 
Degree of substitution:

$$
\begin{aligned}
\mathrm{DS} & =\frac{0.162 \times \mathrm{B}}{1-0.08 \times \mathrm{B}} \\
\mathrm{B} & =\frac{0.1 \times \mathrm{b}}{\mathrm{G}}
\end{aligned}
$$

where, $b$ is (in $\mathrm{mL}$ ) $0.1 \mathrm{~N}$ sulphuric acid volume and $\mathrm{G}$ is pure $\mathrm{CMC}$ mass in grams.

\subsubsection{Moisture content and ash content}

Moisture content was determined at $105^{\circ} \mathrm{C}$ after $2 \mathrm{~h}$ in an oven. [15]. Also, the ash amount was estimated by igniting the sample, at 580$600^{\circ} \mathrm{C}$, in a muffle furnace $[16]$.

\subsubsection{CMC content}

Exactly $1.5 \mathrm{~g}$ of CMC was added to $100 \mathrm{~mL}$ of $80 \%$ aqueous methanol solution. This mixture was stirred, kept for $10 \mathrm{~min}$. and filtered. The cake was washed with $100 \mathrm{~mL} 80 \%$ fresh aqueous methanol and dried to obtain pure CMC [17]. The CMC content was calculated as follows:

$$
\text { CMC content }(\%)=\frac{W}{\mathrm{~W}_{0}} \times 100
$$

where $W_{0}(g)$ is sample weight before washing and $W(g)$ is washed sample weight .

\subsubsection{Solubility determination}

Na-CMC solubility method is based on dissolving a sample in water, followed by this solution filtration through filter funnels.

The filter funnels are dried to constant weight for 2 hours at $a 105^{\circ} \mathrm{C}$ temperature, followed by cooling in a desiccator to room temperature.
$700 \mathrm{~g}$ of $\mathrm{Na}-\mathrm{CMC}$ solution is prepared with $0.1 \%$ mass fraction in absolutely dry technical product terms.

The dissolution is carried out for at least 2 hours with stirring with a propeller-type mixer.

The resulting solution is settled for 1.0-1.5 hours to settle insoluble particles and filtered through a filter funnel on a water-jet pump, while the precipitate is quantitatively transferred to the filter. The residue on the filter is washed with $150-200 \mathrm{ml}$ distilled water with stirring with a glass rod, then washed with $10 \mathrm{ml}$ ethyl alcohol, and then dried to constant weight $\left(\mathrm{m}_{2}\right)$ at a $105^{\circ} \mathrm{C}$ temperature.

$\mathrm{Na}-\mathrm{CMC}$ solubility in water $(\mathrm{Xi})$ in percent is calculated by following formula:

$$
\mathrm{X}_{\mathrm{i}}=\frac{\left(\mathrm{m}_{1}-\mathrm{m}_{2}\right)}{\mathrm{m}_{1}} \cdot 100
$$

where $m_{1}$ is Na-CMC sample weight in absolutely dry matter terms, $\mathrm{g}$;

$m_{2}$ is filter cake, dried to constant weight mass, g.

\subsubsection{Infrared spectroscopic analysis}

$\mathrm{Na}-\mathrm{CMC}$ infrared spectra samples are recorded on a SPECORD instrument in $4000 \mathrm{~cm}^{-1}-400 \mathrm{~cm}^{-}$ ${ }^{1}$ range.

\section{RESULTS AND DISCUSSION}

We have investigated various cellulosecontaining raw materials carboxymethylation possibilities by the suspension method under the same reaction conditions. The original cellulose samples quality indicators are shown in table 1. 


\section{Table 1}

Cellulose-containing raw materials initial samples qualitative indicators

\begin{tabular}{|c|c|c|c|c|c|c|c|c|}
\hline № & Cellulose & Particle & DP & $\begin{array}{c}\alpha- \\
\text { samples } \\
\text { size, } \\
\mu \mathrm{m}\end{array}$ & $\begin{array}{c}\text { White- } \\
\text { ness, } \\
\text { e }\end{array}$ & $\begin{array}{c}\text { Humid } \\
\text { ity, } \%\end{array}$ & $\begin{array}{c}\text { Ash mass } \\
\text { fraction, \% }\end{array}$ & $\begin{array}{c}\text { Degre } \\
\text { e of } \\
\text { crystal } \\
\text { linity \% }\end{array}$ \\
\hline 1 & CC-1 & 1110 & 2700 & 98,0 & 88 & 7,2 & 0,08 & 62 \\
\hline 2 & CC-2 & 780 & 1920 & 98,3 & 91 & 7,0 & 0,06 & 63 \\
\hline 3 & CC-3 & 600 & 1400 & 99,2 & 90 & 7,8 & 0,09 & 65 \\
\hline 4 & PC-1 & 490 & 920 & 98,2 & 89 & 7,6 & 0,09 & 54 \\
\hline 5 & PC-2 & 320 & 670 & 98,5 & 90 & 7,5 & 0,08 & 38 \\
\hline 6 & PC-3 & 210 & 620 & 98,3 & 90 & 7,9 & 0,08 & 22 \\
\hline 7 & MCC-1 & 290 & 340 & 99,0 & 88 & 8,0 & 0,07 & 78 \\
\hline 8 & MCC-2 & 150 & 320 & 98,9 & 89 & 6,3 & 0,05 & 81 \\
\hline 9 & MCC-3 & 90 & 290 & 99,3 & 90 & 7,1 & 0,08 & 84 \\
\hline 10 & MCC-4 & 44 & 260 & 99,1 & 90 & 6,8 & 0,06 & 88 \\
\hline
\end{tabular}

The etherification reaction was carried out according to the well-known suspension method $[18,19]$ in ethyl alcohol medium. For this, $20 \mathrm{ml}$ ethyl alcohol is added to $10 \mathrm{~g} \mathrm{MCC}$ or PC with vigorous stirring for 20 minutes, followed by $30 \mathrm{ml}$ inclusion of $40 \%$ sodium hydroxide aqueous solution. Then, $25 \mathrm{ml}$ ethyl alcohol containing $11,0 \mathrm{~g}$ dissolved MCAA was gradually added to the mixture, and the mixture was stirred for 3 hours at $700 \mathrm{C}$. The reaction product is filtered off, washed with ethyl alcohol and dried to constant weight at 800 C temperature .

In Uzbekistan organic solvents production absence, such as isopropyl alcohol (IPA), benzene, toluene and others, hinders CMC production suspension technology

development. The special grade suspension technology can be mastered in the republic using ethyl alcohol technical grades. At present, the republic has mastered ethyl and methyl alcohol industrial production, which can be used in CMC production by suspension method. Thus, we used ethyl alcohol as an organic solvent for comparative carboxymethylation of cotton cellulose (CC), PC and MCC samples.

The suspension carboxymethylation results of cellulose-containing raw materials samples of 
The American Journal of Applied Sciences

various nature and dispersion under the same conditions are presented in table 2.

Table 2

CMC samples quality indicators

\begin{tabular}{|c|c|c|c|c|c|}
\hline \multirow{2}{*}{ № } & \multirow{2}{*}{$\begin{array}{c}\text { Initial } \\
\text { celluloses }\end{array}$} & \multicolumn{4}{|c|}{ CMC qualitative indicators } \\
\cline { 3 - 6 } & CC-1 & 1410 & 0,61 & 102,4 & Solubility, \% \\
\hline 1 & CC-2 & 980 & 0,63 & 108,5 & 91,2 \\
\hline 2 & CC-3 & 870 & 0,68 & 119,2 & 97,4 \\
\hline 3 & PC-1 & 840 & 0,98 & 147,4 & 99,0 \\
\hline 4 & PC-2 & 590 & 1,07 & 156,5 & 100 \\
\hline 5 & PC-3 & 530 & 1,14 & 161,3 & 100 \\
\hline 6 & MCC-1 & 310 & 0,77 & 135,9 & 100 \\
\hline 7 & MCC-2 & 290 & 0,81 & 153,7 & 100 \\
\hline 8 & MCC-3 & 230 & 0,88 & 168,0 & 100 \\
\hline 9 & MCC-4 & 220 & 0,90 & 187,8 & 100 \\
\hline 10 & & & & & 100 \\
\hline
\end{tabular}

As seen in table 2, the reduced particle size of the cellulose samples provides a larger surface area, which increases the interaction likelihood between the reagent and the cellulose. This makes it possible to increase the reaction rate, as well as the yield and DS of CMC [20]. An increase in DS means that each cellulose anhydroglucose unit (AGU) reacts with alkylating agents, thereby increasing the resulting carboxymethyl cellulose mass.

High value DS of CMC obtained by the suspension method is explained by the low water content in the reaction medium, as a result of which MCAA hydrolysis side reaction rate significantly decreases in the aqueous organic medium, as a result, the etherifying agent consumption useful coefficient increases. CMC solubility index high value samples is apparently explained by high value

DS of CMC, in particular, its compositional homogeneity and low values of the DP.

The CMC yield also increases with increasing DS. This phenomenon suggests that at a higher DS, more MCAA molecules are replaced by the a-cellulose polymer. This substitution reduces MCAA ability to react with $\mathrm{NaOH}$ with the formation of by-products [21]. The use of alcohol as a solvent in the carboxymethylation reaction ensures the miscibility and availability of the etherifying agent for the reaction rather than for the formation of glycolate [22]. 
It was found that the solubility of CMC in water increases with a decrease in the particle size of the feedstock. Under this standard condition, the CMC solubility samples obtained from PC and $M C C$ reaches a maximum value, which requires determining the optimal reaction conditions for the carboxymethylation of PC and MCC by the suspension method.
We have investigated reaction conditions effect of CC, MCC, and PC carboxymethylation on the physicochemical parameters of the obtained CMC samples and determined the optimal conditions for obtaining highly substituted CMC (PAC) samples from MCC and $\mathrm{PC}$ by the suspension method in ethanol.

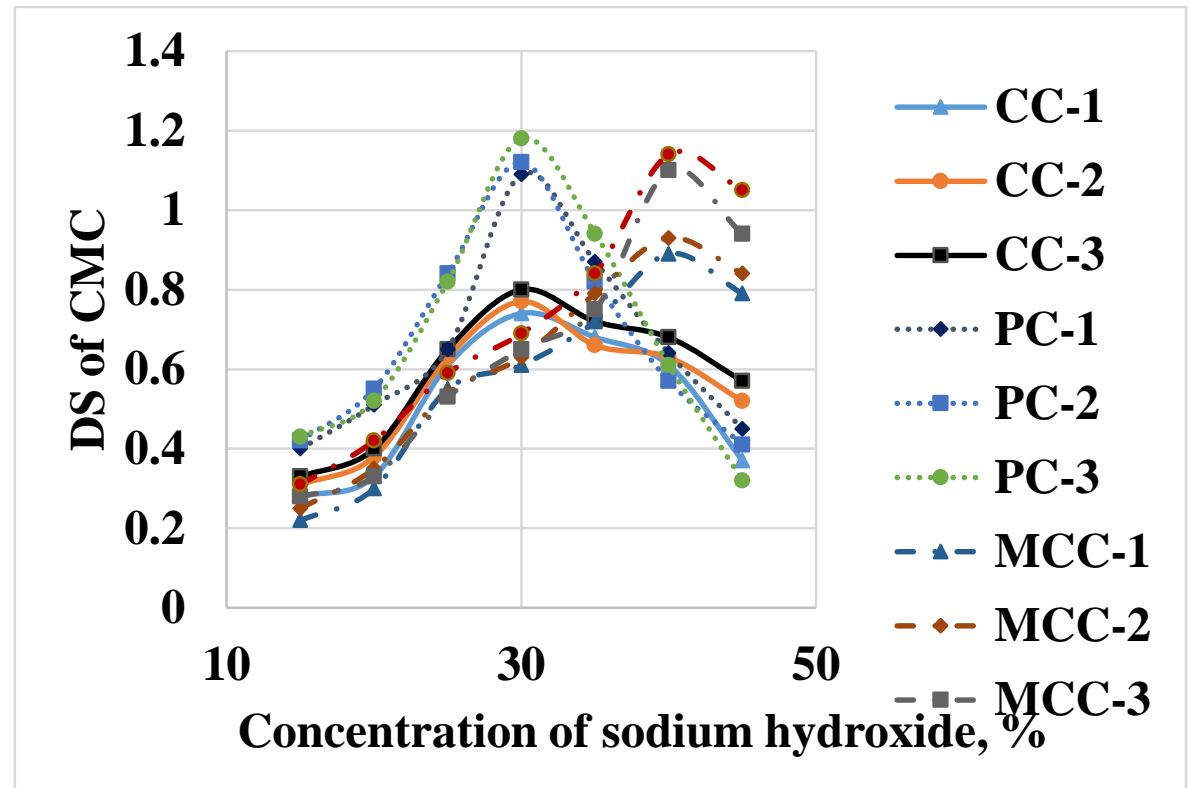

Figure 3.13 Dependence of DS of CMC on sodium hydroxide concentration

By increasing the concentration of sodium hydroxide DS of CMC passes through a maximum and shows the highest DS product reaches when the alkaline concentration for $C C$ is $35 \%$, for PC is $30 \%$ and for MCC is $40 \%$, respectively. Further increases in alkaline concentration lead to a decrease in DS of CMC, which indicates an increase in the rate of side reactions like hydrolysis of MCAA.

In fig. 3.14 presents data on the study of the effect of the reaction temperature of carboxymethylation on DS of CMC. 


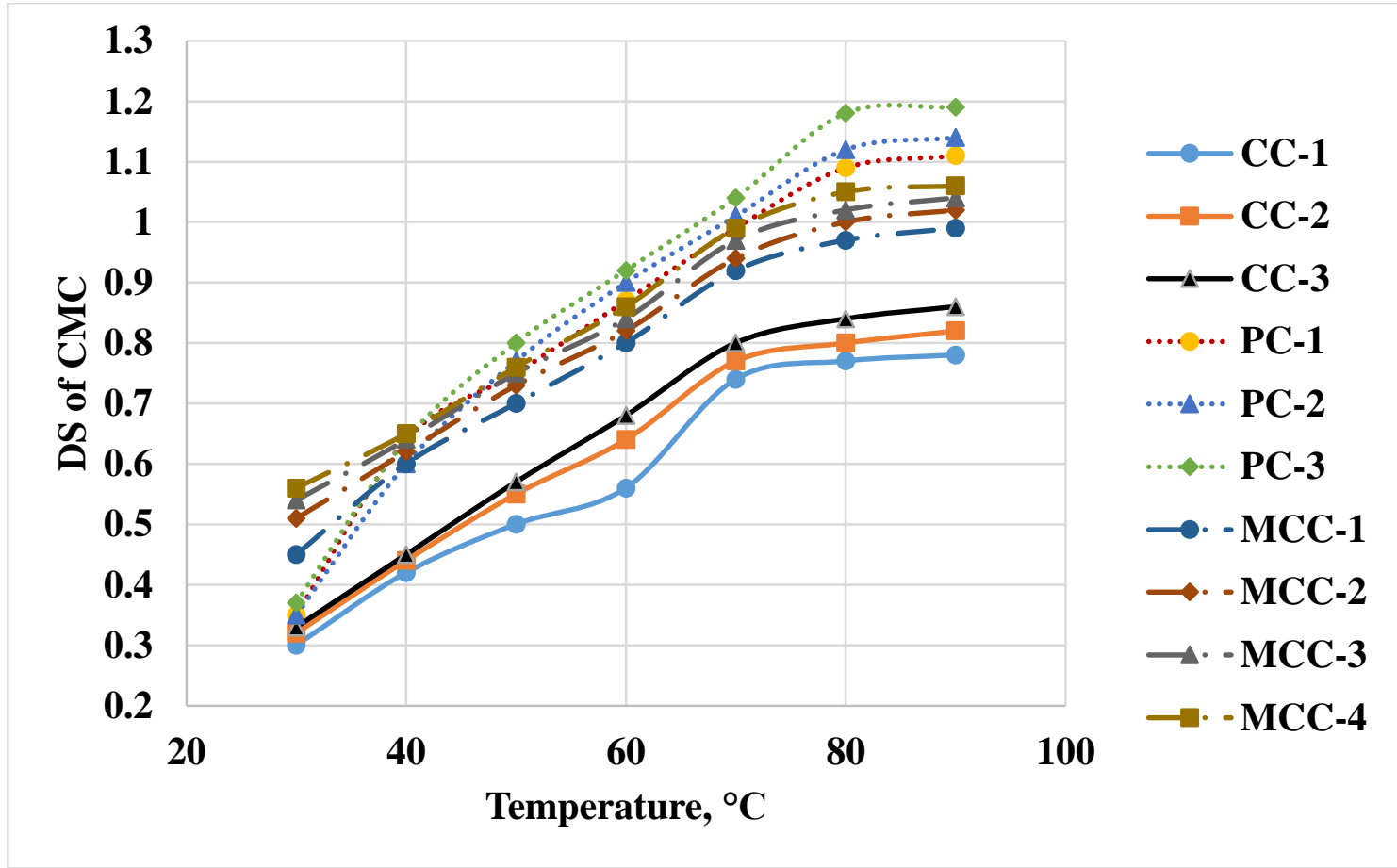

Figure 3.14 Dependence of DS of CMC on reaction temperature

The carboxymethylation reaction is exothermic. During the reaction, heat is released and the temperature of the mass spontaneously increases, which is required for the start of the reaction heat energy [23]. In this case, the change in the degree of substitution of Na-CMC is of the same character as the change in temperature. As seen from fig. 3.14, an increase in the reaction temperature of carboxymethylation to $800 \mathrm{C}$ DS of CMC reaches an optimal value, with further increases in the temperature of the reaction mass, the DS of the product does not change.

The reaction completion also depends on the carboxymethylation duration, which results are shown in fig. 3.15. 


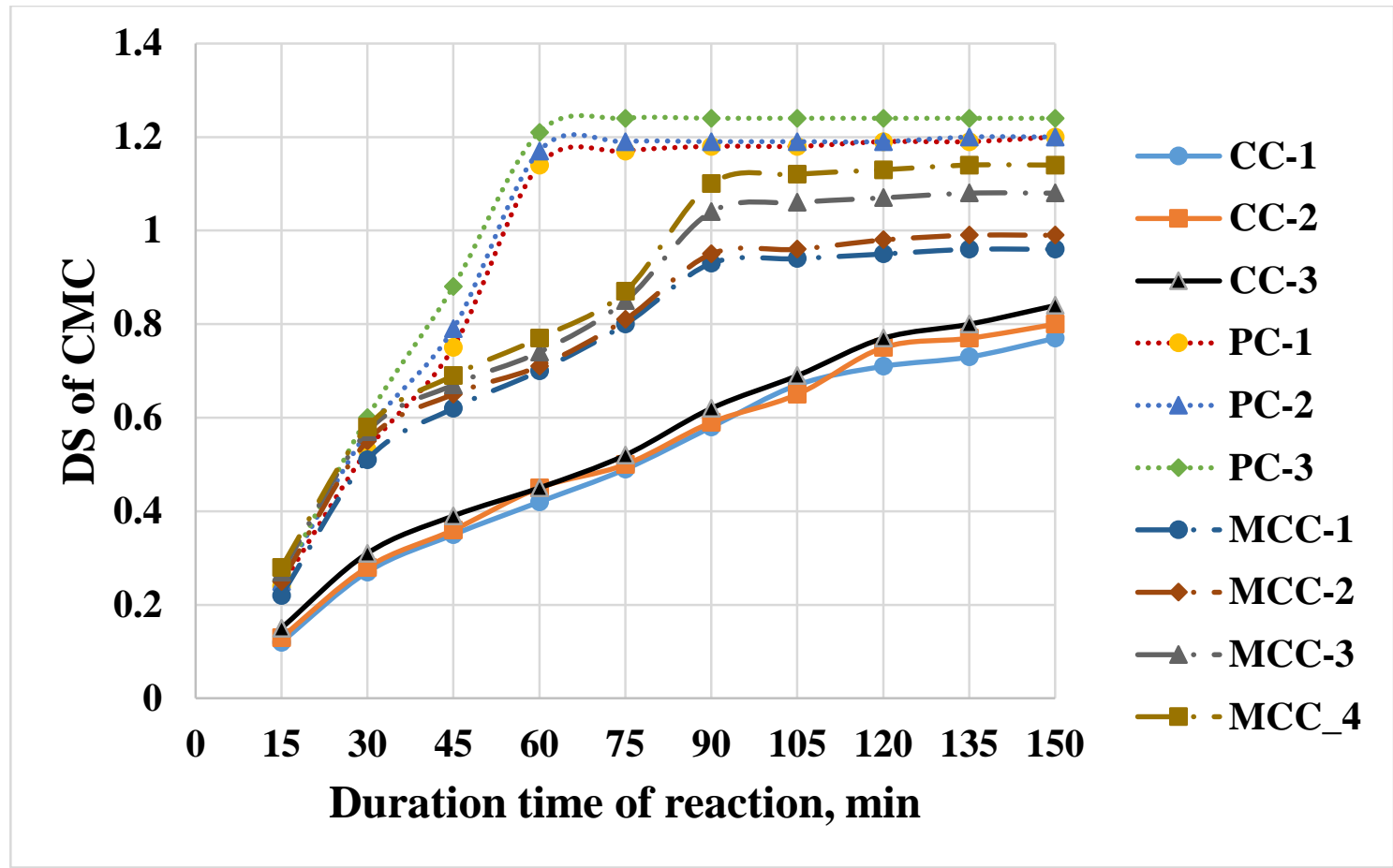

Figure 3.15 Dependence of DS of CMC on the carboxymethylation duration

As can be seen from Fig. 3.15, DS of CMC maximum value reaches 150,60 and 90 minutes when carrying out the carboxymethylation reaction, at $80^{\circ} \mathrm{C}$, with an alkaline concentration for $35 \%, 30 \%$ and $40 \%$ CC, PC and MCC, respectively.
Figure 3.16 shows carboxymethylation reaction results of CC, MCC and PC alkaline samples, which were obtained by treating them with 35 , 30 and $40 \%$ sodium hydroxide solutions, followed by etherification with various concentrations MCCA. 


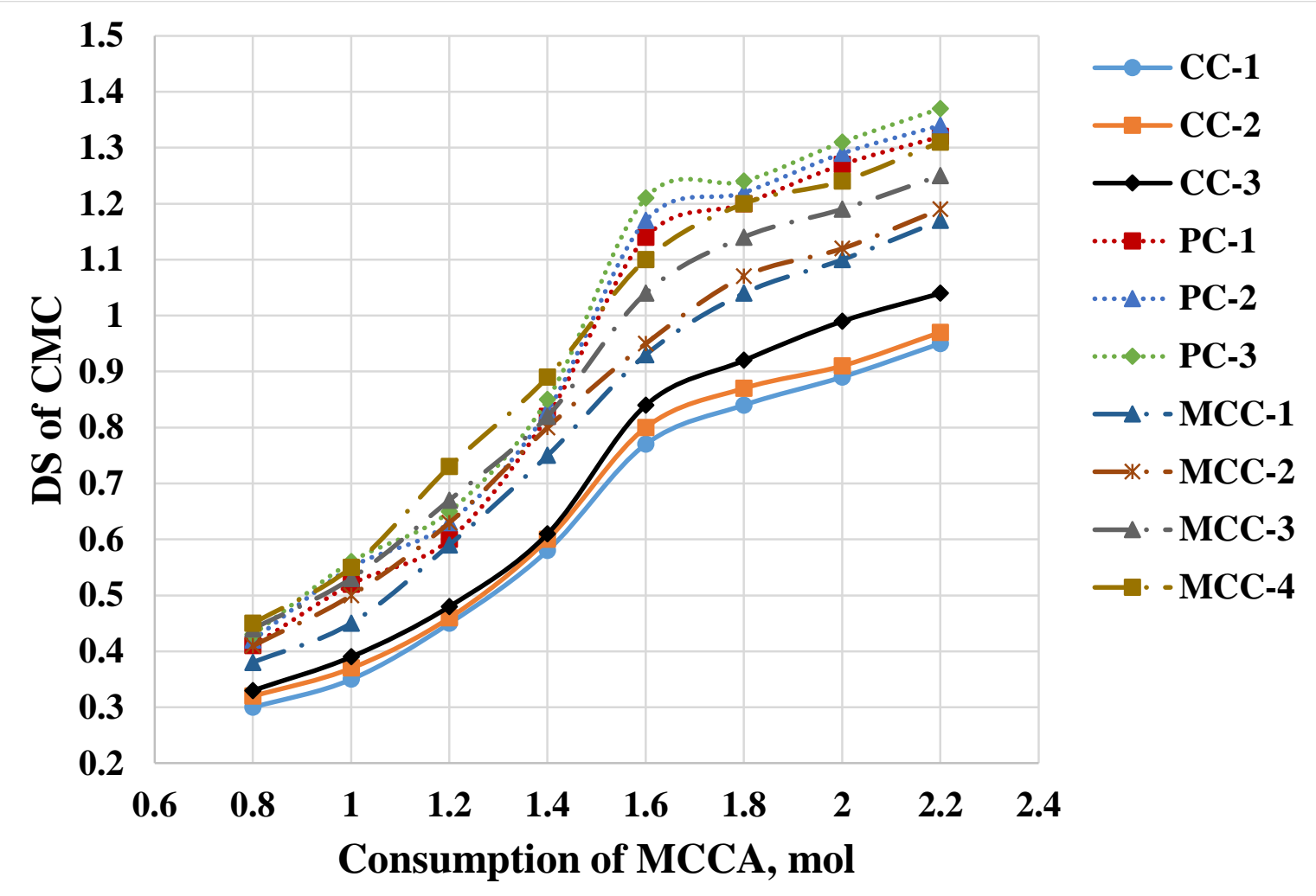

Figure 3.16 Dependence of CMC DS on MCAA consumption

It can be seen that with an increase in MCAA consumption to 2.2 mole relative to cellulose anhydroglucose unit increasing and effective DS of CMC reaches at 1.6 mole of MCAA consumption.
Next, we carried out a study to establish the DS of $C M C$, which ensures complete dissolution in water, depending on their particle size. The results are shown in fig. 3.17. 


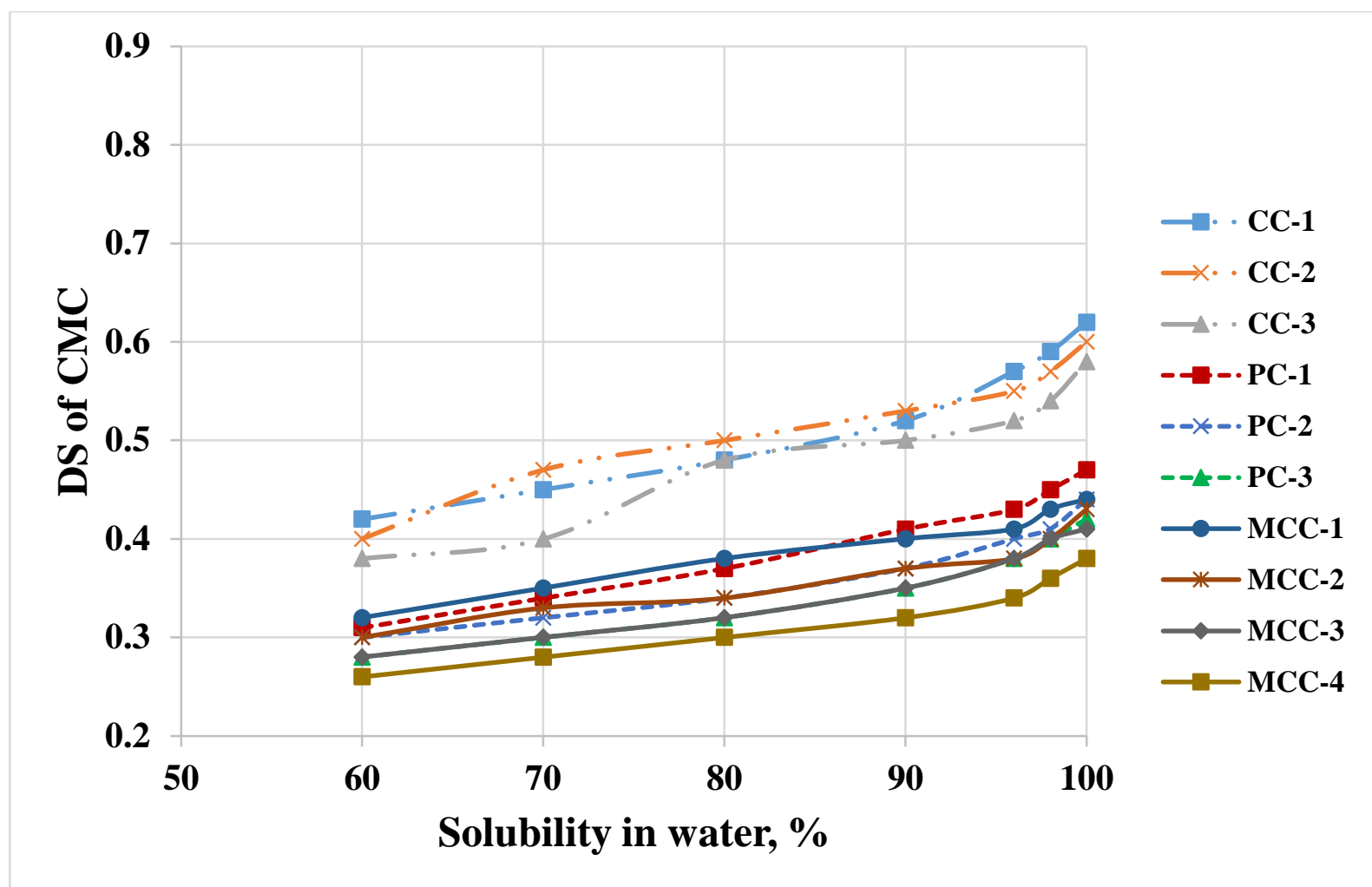

Figure 3.17 Dependence of solubility of CMC in water on its DS

As can be seen from fig. 3.17, the CMC solubility samples obtained from CC, PC and MCC reaches its maximum value at their DS-0.580.62 , DS-0.42-0.47 and DS-0.38-0.44 respectively.

The relatively DS low values of the watersoluble CMC samples can be explained by particle size and macromolecule length low values of the initial samples. With an increase in the dispersion degree, i.e. a decrease in the particle size and a decrease in the DP of the initial samples increases their swelling degree in alkaline solutions, which contributes to a more efficient destruction of intermolecular hydrogen bonds. Also, the reduced particle size of the initial cellulose samples provides a large surface area, which increases the alkylating agent availability - MCAA with a cellulose elementary unit. Due to this, the carboxymethylation reaction rate increases and DS of product increases. Short macromolecules enable CMC dissolution in water despite its low DS.

On the basis of the data obtained, the optimal conditions for obtaining $C M C$ by the suspension method have been established, which are shown in Table 3.5. 
Table 3.5

Optimal conditions for obtaining water-soluble CMC based on CC, PC and MCC by the suspension method

\begin{tabular}{|c|c|c|c|}
\hline $\begin{array}{c}\text { Parameters } \\
\text { Sodium hydroxide concentration, } \\
\%\end{array}$ & 35 & PC & MCC \\
\hline Temperature, ${ }^{\circ} \mathrm{C}$ & 80 & 80 & 80 \\
\hline Reaction time, min & 150 & 60 & 90 \\
\hline MCAA consumption at DS-0.8-0.9, & 1,4 & 0,8 & $0,7-0,8$ \\
\hline mole & & & $0,38-0,44$ \\
\hline DS of CMC with completely & $0,58-0,62$ & $0,42-0,47$ & \\
\hline
\end{tabular}

IR-spectra of water-soluble CMC samples are shown in Figure 3.18.

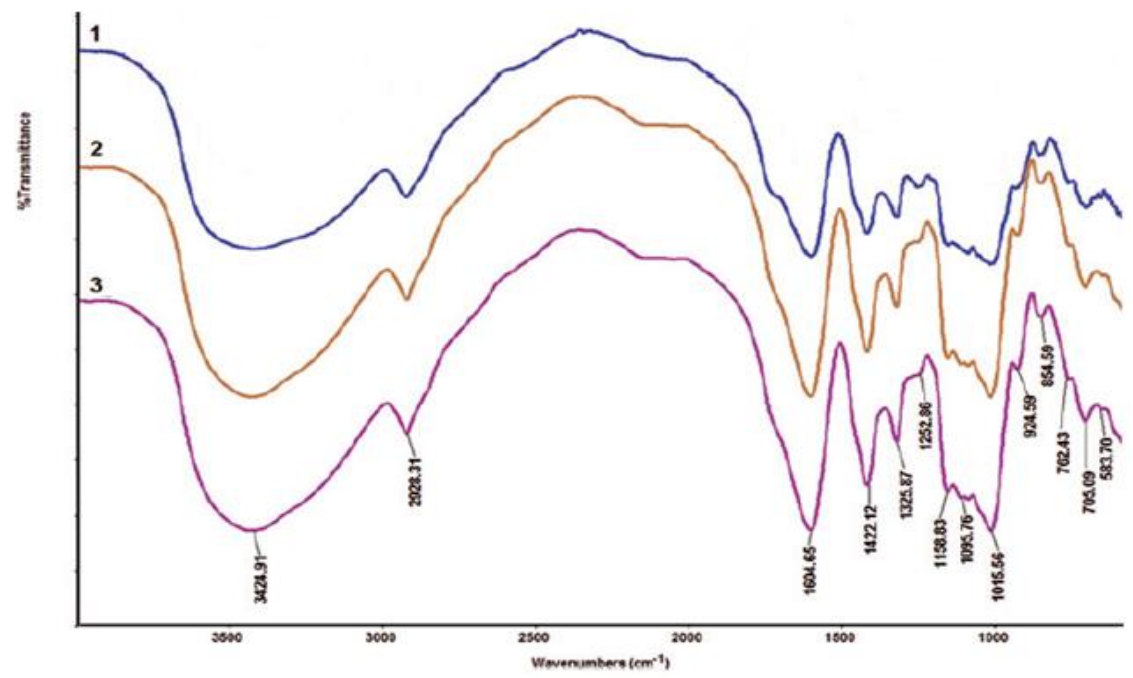

Figure: 3.18. IR-spectra of CMC samples 
The CMC samples IR-spectrum shows that the polymer carboxymethyl and hydroxyl groups are in the absorption bands at 1600-1605 cm-1, 1422 и $1325 \mathrm{~cm}-1$, respectively. According to the data presented, absorption bands at $1605 n$ $1422 \mathrm{~cm}-1$ correspond to two carboxyl and methyl functional CMC groups [24]. Additional absorption bands at 2152 и 2376 cm-1 can be associated with their by-products presence in CMC - reactions or a combined bond of CMC with water [25]. Obviously, a broad absorption band at $3424 \mathrm{~cm}-1$ и $2928 \mathrm{~cm}-1$ respectively characterizes with hydroxyl (-OH) and methylene $(\mathrm{C}-\mathrm{H})$ asymmetric vibrations groups. These bonds types have a mobile terminal hydrogen atom. This kind bonds oscillation in a molecule is due to a slight effect from the rest of the molecule. It can be argued that in such compounds there is a set of energetically unequal hydrogen bonds [26]. Strong vibrations at 1605 cm-1 confirmed carboxymethyl anion carbonyl group presence $(-C=O)$. The wide band absence in 2500-2800 $\mathrm{cm}-1$ region and a high value of the carboxyl group stretching vibrations band position indicate that carboxyl groups content is not at a high level, and they do not form hydrogen bonds with each other.

The absorption bands at 1422 and $1325 \mathrm{~cm}-1$ refer to the - $\mathrm{CH}_{2}$-group and free hydroxyl groups $(-\mathrm{OH})$, respectively.

Absorption in the $158-1095 \mathrm{CM}-1$ region refers to $\mathrm{CO}$ groups stretching vibrations. With an increase in the DS of CMC, the bands intensity decreases, characterizing the in-plane bending vibrations of hydroxyl groups. A band appears, characterizing the $\mathrm{C}-\mathrm{O}-\mathrm{C}$ vibrations ether bond. $[27,28]$.

As the studies results carried out, it was obtained and characterized highly substituted CMC samples based on MCC and PC.

It is known that highly substituted CMC (PAC) production in the world is carried out by multistage feedstock carboxymethylation, which leads to an increase in final product cost due to an increase in chemical reagents and energy consumption.

It is shown that PC use as a feedstock in onestage carboxymethylation makes it possible to obtain PAC highly substituted samples, which are a valuable reagent for the oil and gas industry, in drilling and other fluids in oil and gas production.

Thus, the optimal conditions for water-soluble CMC synthesis samples based on CC, PC and MCC were established, as a reaction result, low-substituted, completely soluble CMC samples in water with DS-0.58, 0.42 and 0.38 respectively by suspension method. Also it was obtained highly substituted samples of CMC with DS-1.37, as called "polyanionic cellulose".

\section{CONCLUSION}

As the studies result carried out, it was obtained and characterized low and highlysubstituted CMC samples based on CC, MCC and PC.

It is known that obtaining highly substituted CMC i.e. polyanionic cellulose (PAC) is carried out by multi-stage feedstock carboxymethylation, which leads to an increase in final product cost due to an increase in chemical reagents and energy consumption. It is shown that PC use as a feedstock for onestage carboxymethylation makes it possible to obtain PAC samples, which are a valuable reagent for the oil and gas industry, in the drilling and other fluids in oil and gas productions.

\section{REFERENCES}

1. V.A. Bondar, V.V. Kazansev, "State of cellulose ethers production", Cellulose ethers and starch: synthesis, properties, application. Materials of the Xth anniversary of Russian sci. and tech. conf. with int. part., Suzdal, pp. 9-26, 2003. 
2. A.A. Ibrahim, A.M. Adel, Z.H. El-Wahab, M.T. Al-Shemy, "Utilization of carboxymethyl cellulose based on bean hulls as chelating agent, Synthesis, characterization and biological activity", Carbohydrate Polymers, vol. 83, no. 4, pp. 94-115, 2011..

3. M.A. Zeenat and et al., "Polymeric Cellulose Derivative: CarboxymethylCellulose as useful Organic Flocculant against Industrial Waste Waters" Int. Journal of Advancements in Research \& Technology, vol. 2, no. 8, pp. 14-20, 2013.

4. Cheng et al., "Energy consumption and morphological development of eucalyptus alkaline peroxide mechanical pulp by carboxymethyl cellulose - assisted refining" BioResources, vol. 8, no. 2, pp. 2173-2185, 2013.

5. M. Mohkami, M. Talaeipour "Investigation of the chemical structure of carboxylated and carboxymethylated fibers from waste paper via XRD and FTIR analysis", BioResources, vol. 2, no. 6, pp. 1988-2003, 2011.

6. H. Xiaojia, W. Shaozu, F. Dongkang, N. Jinren, "Preparation of sodium carboxymethyl cellulose from paper sludg" J.Chem. Technol. Biotechnol., vol. 84, no.3, pp. 427-434, 2009.

7. Z. Guo, R. Xing, S. Liu, Z. Zhong, "Synthesis and hydroxyl radicals scavenging activity of quaternized carboxymethyl chitosan", Carbohydrate Polymers, vol. 73, pp. 173-177, 2008.

8. T. Heinze, K. Pfeiffer, "Studies on the synthesis and characterization of carboxymethyl celluloses", DieAngew. Makromol. Chem., vol. 266, pp. 37-45, 1999.

9. Sh.A. Yuldoshov, A.I. Shukurov, A.A. Sarymsakov, S.Sh. Rashidova, "Preparation of carboxymethyl cellulose solutions by freezing-thawing", Universum: Chemistry and Biology, vol. 23, no. 5, pp. 1-9, 2016.

10. N.I. Nisa, S. Toto, S. Unang, "Synthesis and characterization of polyanionic cellulose from kapok plant (Ceiba petandra) fibres as a drilling fluid additive in oil and gas exploration" Proceedings of the 2nd International Seminar on Chemistry, Jatinangor, pp. 234-239, Nov. 24-25, 2011.

11. A.F. Maas, B.V. Noviant, O.G. Isaenko, "Differences between sodium carboxymethyl cellulose and polyanionic cellulose (PAC)", Development, production and application of chemicals for the oil and gas industry. Materials Russ. scientific and practical. conf., pp. 226-229, 2002.

12. A.A. Sarymsakov, Medium- and lowsubstituted CMC: production, properties and application. Tashkent: Fan, pp. 5-14, 2005.

13. A. Bono, P. H. Ying, F. Y. Yan, C. L. Muei, R. Sarbatly, D. Krishnaiah, "Synthesis and characterization of carboxymethyl cellulose from palm kernel cake", Adv. Nat. Appl. Sci. no. 3, pp. 5-11, 2009.

14. S.Veronica, K. Goran, G.Ulf, "The influence of the solvent system used during manufacturing of CMC", Cellulose, no. 13, pp. 705-712, 2006.

15. Md. I. H. Mondal, A. B. M. F. Alam, "Utilization of cellulosic wastes in textile and garment industries. 2. Synthesis and characterization of cellulose acetate from knitted rag", J. Polym. Environ, no. 21, pp. 280-285, 2013.

16. ASTM. Standard Test Method for Ash in pulp, paper and paper product, American Society for Test Material, Philadelphia, USA, D5, pp. 86-97, 2002.

17. H. Togrul, N. Arslan, "Production of carboxymethyl cellulose from sugar beet pulp cellulose and rheological behaviour of carboxymethyl cellulose", Carbohydr. Polym., no. 54, pp. 73-82, 2003.

18. Sh.A. Yuldoshov, A.A. Sarymsakov, S.Sh. Rashidova, "Investigating of of synthesizing possibility of low-viscosity carboxymethyl cellulose based on microcrystalline cellulose", Institute of Plant Chemistry named after acad. S.Yu. 
Yunusov UzAS, Tashkent, March 12, pp. 191, 2015.

19. Sh.A. Yuldoshov, A.I. Shukurov, A.A. Sarymsakov, "Suspension technology of production of carboxymethyl cellulose and a method of its purification", Bioorganic chemistry in solving urgent problems of healthcare and agriculture, Tashkent, Oktob, 15-16, pp. 67-72, 2016.

20. N. Olaru, L Olaru, "Influence of organic dilutenson cellulose carboxymethylation" Macromol. Chem. Phys., no. 202, pp. 207211, 2001.

21. N. Olaru, L. Olaru, A. Stoleriu, D. Timpu, "Carboxymethylcellulose synthesis in organic media containing ethanol and or acetone" J. App. Polym. Sci., no. 67, pp. 481-486, 1997.

22. F.R. deAbreu, P. Sergio, C. Filho, "Characteristics and properties of carboxymethyl chitosan" Carbohydrate Polymers, no. 75, pp.214-221, 2009.

23. A. Bono, P.H. Ying, F.Y. Yan, C.L. Muei, R. Sarbatly, D. Krishnaiah, "Synthesis and Characterization of Carboxymethyl Cellulose from Palm Kernel Cake", Adv. Natl. Appl.Sci. vol. 1, no. 3, pp. 5-11, 2009.

24. D.R. Biswal, R.P. Sing, "Characterisation of carboxymethyl cellulose and polyacrylamide graft copolymer", Carbohydr. Polym., no. 57, pp. 379-387, 2004.

25. J. Wang, P. Somasundaran, "Adsorption and conformation of carboxymethylcellulose at solid-liquid interfaces using spectroscopic, AFM and allied techniques", Journal of Colloid and Interface Science, no. 291, pp. 75-83, 2005.

26. G.M. Thomas, E.M. Paquita, J.P. Thomas, "Cellulose ethers", Encyclopedia of polymer science and technology, New York: Wiley, -pp. 221, 2002.

27. R.G.P. Viera, G.R. Filho, R.M.N. Assuncao, C.S. Meireles, J.G. Vieira, G.S. Oliveira, "Synthesis and characterization of methylcellulose from sugar cane bagasse cellulose", Carbohydr. Polym., vol. 2, no. 67, pp. 182-189, 2007.

28. D.R. Biswal, R.P. Singh, "Characterization of carboxymethyl cellulose and polyacrylamide graft copolymer", Carbohydr. Polym., no. 57, pp. 379-387, 2004. 